\title{
Necrotizing Tracheitis Secondary to Corynebacterium Species Presenting With Central Airway Obstruction
}

\author{
Jorge Guerrero MD, Pavan Mallur MD, Erik Folch MD MSc, Colleen Keyes MD MPH, \\ Isaac E Stillman MD, Sidhu P Gangadharan MD, and Adnan Majid MD
}

\begin{abstract}
Pseudomembranous necrotizing tracheitis is a rare cause of life-threatening central airway obstruction. Patients present with hoarseness, cough, dyspnea, wheezing, stridor, and occasionally with respiratory failure. Diagnosis requires a high index of suspicion, and should be confirmed with a comprehensive bronchoscopy and biopsy. For patients with signs and symptoms of airway obstruction, a therapeutic rigid bronchoscopy with mechanical debridement is recommended. We present a rare case of necrotizing tracheitis secondary to Corynebacterium species, which caused life-threatening airway obstruction in an adult. Key words: central airway obstruction; Corynebacterium; necrotizing tracheitis; rigid bronchoscopy. [Respir Care 2014;59(1):e5-e8. () 2014 Daedalus Enterprises]
\end{abstract}

\section{Introduction}

Pseudomembranous necrotizing tracheitis is a rare cause of life-threatening central airway obstruction. Pseudomembrane airway disease is due to either an infectious or non-infectious process. The most common infectious etiologies include Corynebacterium diphtheria, Corynebacterium pseudodiphtheriticum, Staphylococcus aureus, Bacillus cereus, Aspergillus species, and Haemophilus influenzae..$^{1-7}$ Among the non-infectious etiologies, endotracheal intubation is the most common. ${ }^{8}$ Other reported etiologies include smoke inhalation, Stevens-Johnson syndrome, paraquat ingestion, and autoimmune disorders such as Crohn disease. ${ }^{9-12}$ Patients will present with hoarseness, cough, dyspnea, wheezes, stridor, and, occasionally, respiratory failure. Diagnosis requires a high index of sus-

\footnotetext{
The authors are affiliated with the Division of Thoracic Surgery and Interventional Pulmonology, with the exception of Dr Mallur, who is affiliated with the Department of Otolaryngology, and Dr Stillman, who is affiliated with the Department of Pathology, Beth Israel Deaconess Medical Center, Harvard Medical School, Boston, Massachusetts.

The authors have disclosed no conflicts of interest.

Correspondence: Adnan Majid MD, Division of Thoracic Surgery and Interventional Pulmonology, Beth Israel Deaconess Medical Center, 185 Pilgrim Road, Deaconess 201, Boston MA 02215.
}

DOI: $10.4187 /$ respcare. 02150 picion, and should be confirmed with a comprehensive bronchoscopic examination and biopsy. For patients with signs and symptoms of airway obstruction, a therapeutic rigid bronchoscopy with mechanical debridement is recommended. We present a rare case of necrotizing tracheitis secondary to Corynebacterium species, which caused life-threatening airway obstruction in an adult.

\section{Case Report}

A 39-year-old woman with morbid obesity, diabetes mellitus, coronary artery disease, and Crohn disease presented with acute shortness of breath and stridor 5 weeks after coronary artery bypass surgery. Her postoperative course was complicated with post-pericardiotomy syndrome (Dressler syndrome), and was treated with dexamethasone, $4 \mathrm{mg}$ intravenously every 8 hours, for at least 10 days. Her shortness of breath progressed to stridor in a matter of hours. Computed tomography showed severe tracheal narrowing, with an airway lumen of $4 \mathrm{~mm}$ (Fig. 1). She was bridged with heliox (70\% helium/30\% oxygen) and underwent emergency rigid bronchoscopy. Endoscopic examination revealed a grossly estimated $95 \%$ obstruction of the proximal trachea, mainly due to the formation of a pseudomembrane. Mechanical debridement uncovered an area of severe tracheal inflammation, with multiple mucosal, plaque-like lesions suggestive of an infectious etiology (Fig. 2). Pathology revealed extensively necrotic soft tissue, cartilage, and bone, with acute and chronic inflam- 


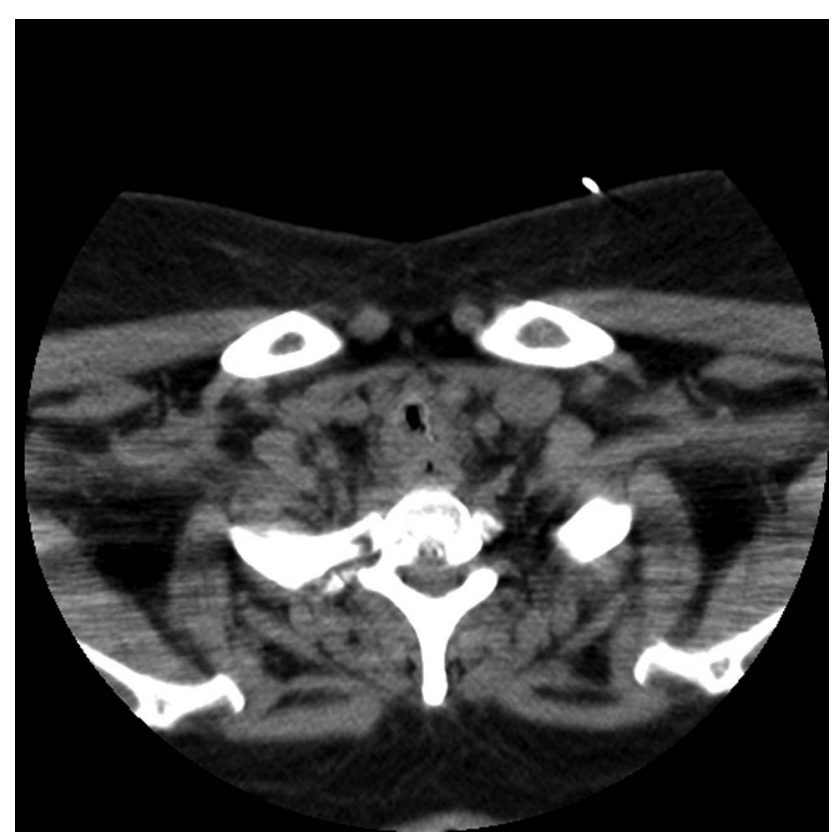

Fig. 1. Computed tomography shows severe tracheal stenosis, with an airway lumen of $4 \mathrm{~mm}$.

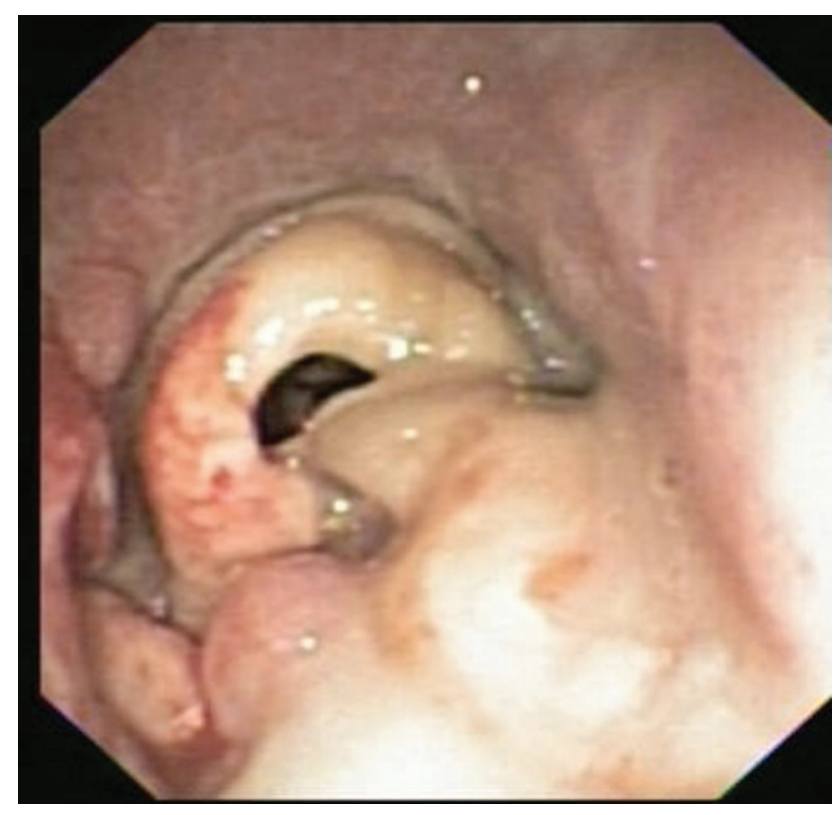

Fig. 2. Bronchoscopy in the subglottic area shows tracheal inflammation suggestive of an infectious etiology.

mation and bacterial overgrowth (Fig. 3A). Tissue Gram stain revealed numerous Gram-positive rods (see Fig. 3B). Biopsies grew Corynebacterium species sensitive to clindamycin. She was discharged with a 2-week course of antibiotic and close follow-up in the out-patient clinic. Unfortunately, she did not return for her follow-up visit, and 3 weeks later presented with acute dyspnea and stridor, and required emergency intubation with a size 5.0

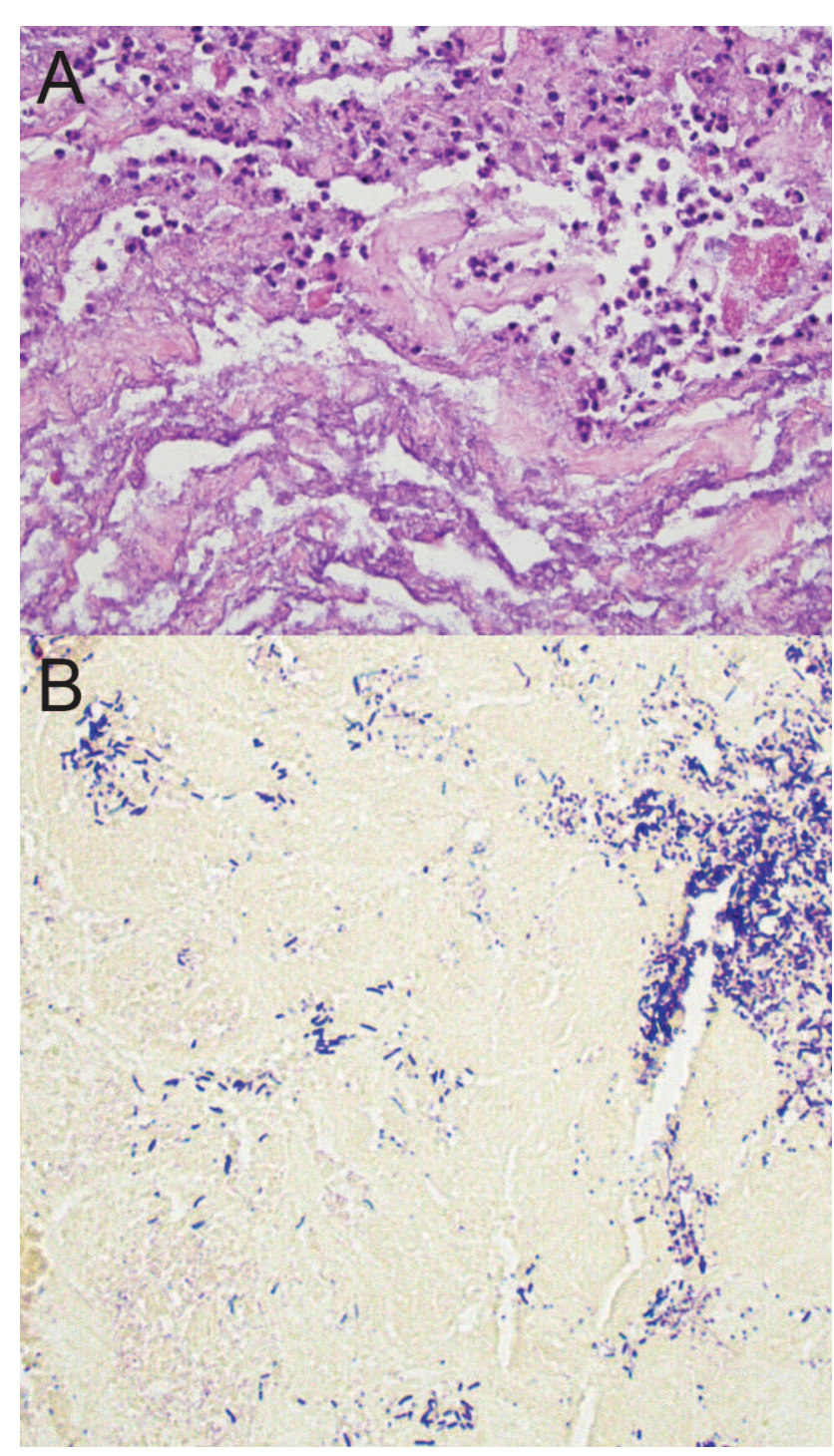

Fig. 3. A: Extensive necrotic tissue with neutrophilic infiltrate and bacterial overgrowth. B: Gram stain reveals numerous Grampositive rods.

endotracheal tube. Flexible bronchoscopy was attempted in an effort to bronchoscopically advance the endotracheal tube past the tracheal stenosis, but this was unsuccessful, given the reduced diameter of the airway. The patient was taken to the operating room for emergency rigid bronchoscopy and was started on imipenem and vancomycin to treat her recurrent infection. Endoscopy showed critical airway obstruction due to complex tracheal stenosis, which started $1.8 \mathrm{~cm}$ from the vocal cords and extended $4 \mathrm{~cm}$ in length. Dilation with the rigid bronchoscope barrel was performed, to $10 \mathrm{~mm}$, with suboptimal response, and the decision was to perform a percutaneous dilation tracheostomy during the same case, guided by rigid bronchoscopy. After the procedure she was admitted to the medical floor 


\section{Necrotizing Tracheitis Secondary to Corynebacterium Species}

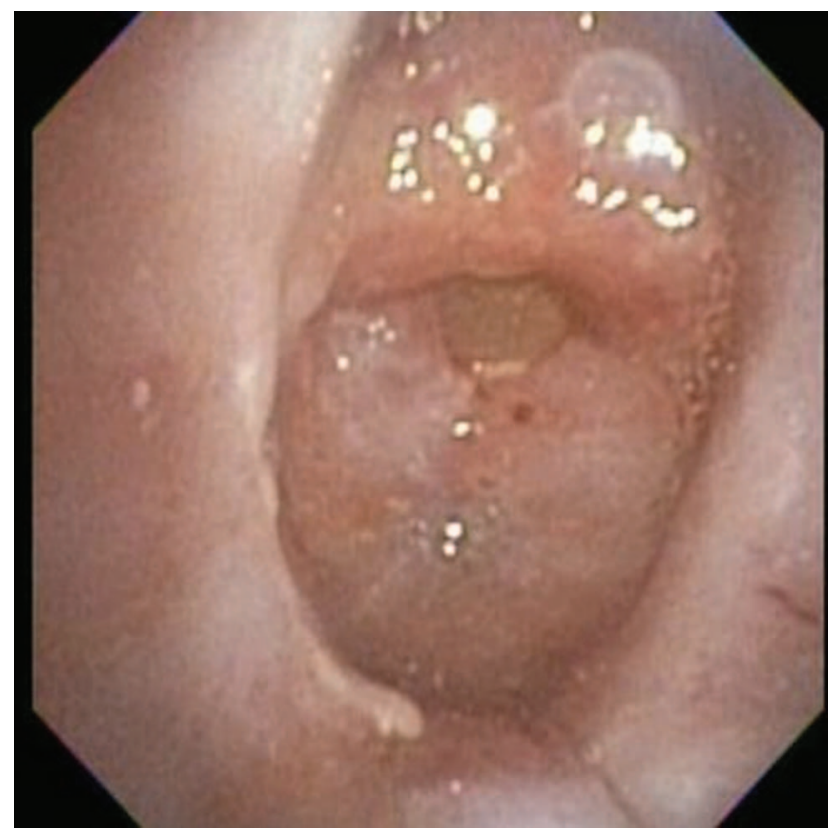

Fig. 4. Bronchoscopy in the subglottic area shows complete central airway obstruction due to tracheal stenosis.

for observation, and was discharged at 72 hours, with scheduled follow-up in 2 weeks.

Eight days later she was readmitted with sudden dyspnea, secondary to mucus plugging of the tracheostomy tube. A repeat flexible bronchoscopy showed complete airway obstruction from progressive granulation tissue formation at the proximal trachea, right above the stoma (Fig. 4). The tracheostomy tube was left in place, and the airway surgeon was consulted. She underwent tracheal resection and reconstruction, which restored complete airway patency, and she no longer required a tracheostomy tube.

\section{Discussion}

Corynebacterium is an aerobic Gram-positive bacillus, widely distributed in nature, and is mostly innocuous. Although it can be an airway colonizer in the immunocompetent patient, it is known to cause a severe form of pseudomembranous necrotizing tracheitis in the immunocompromised host. ${ }^{3}$ Risk factors that have been identified based on the few case reports published in the literature include diabetes mellitus, immunosuppression, COPD, congestive heart failure, and underlying malignancy. ${ }^{1-5}$ The diagnosis of Corynebacterium infectious tracheitis should be confirmed with a comprehensive bronchoscopic examination and biopsy.

Specific antibiotic therapy is warranted once the organism is isolated. One study of the minimum inhibitory concentrations for antibiotics revealed that Corynebacterium pseudodiphtheriticum isolates are susceptible to ampicillin, amoxicillin/clavulanic acid, cefazolin, cefuroxime, ceftazidime, and imipenem. ${ }^{1}$ Another study showed that it is susceptible to beta-lactam antibiotics, clindamycin, vancomycin, and trimethoprim-sulfamethoxazole. ${ }^{2}$ Given the possibility of resistance to antibiotic monotherapy, especially in patients with severe airway involvement, antibiotic combination to achieve synergy would be reasonable to avoid relapse and further complications, as seen with our patient. Ultimately, the choice of antibiotics should be based on organism-specific sensitivities and patient characteristics, including renal function, allergies, and cross-reactivity with other medications.

Alternate etiologies for this presentation should be considered as described previously. Central airway obstruction due to Crohn disease was of particular concern, given the patient's medical history. However, Crohn disease in the airway is typically responsive to steroids or may happen after steroids are withdrawn. ${ }^{12}$ Our patient was on high doses of dexamethasone prior to and during her presentation. Furthermore, the rapid onset of symptoms made Crohn disease less likely to be the etiology and made infection a more plausible cause.

In addition to antibiotic therapy for Corynebacterium tracheitis, patients with signs and symptoms of central airway obstruction need immediate bronchoscopic intervention to restore airway patency. Whenever feasible the patient should be weaned off immunosuppressive medications, and other underlying conditions (eg, diabetes) need to be aggressively controlled. In patients with evidence of severe tracheitis we suggest early bronchoscopic followup, within the first week after the initial intervention, given the concern about early scar formation and recurrent airway obstruction, as in our patient. In patients with recurrent airway obstruction, therapeutic options include tracheostomy, Montgomery T-tube, and airway stent.

For patients who present in respiratory failure, as did our patient, a tracheostomy tube is the best option because it allows for mechanical ventilation and serves as a conduit for suctioning inflammatory debris. Tracheostomy can be a bridge to surgical intervention, or permanent in patients deemed not to be surgical candidates. Due to the need for complex endoscopic multimodality therapy and the potential for complications, these patients are best managed at a center of excellence where an interventional pulmonologist, a laryngologist, and a thoracic surgeon are available.

\section{REFERENCES}

1. Ahmed K, Kawakami K, Watanabe K, Mitsushima H, Nagatake T, Matsumoto K. Corynebacterium pseudodiphtheriticum pathogen: a respiratory tract pathogen. Clin Infect Dis 1995;20(1):41-46.

2. Craig TJ, Maguire FE, Wallace MR. Tracheobronchitis due to 


\section{Necrotizing Tracheitis Secondary to Corynebacterium Species}

Corynebacterium pseudodiphtheriticum. South Med J 1991;84(4): 504-506.

3. Colt HG, Morris JF, Marston BJ, Sewell DL. Necrotizing Tracheitis caused by Corynebacterium pseudodiphtheriticum: unique case and review. Rev Infect Dis 1991;13(1):73-76.

4. Hadfield TL, McEvoy P, Polotsky Y, Tzinserling V, Yaklovev A. The pathology of diphtheria. J Infect Dis 2000;181(Suppl 1):S116S120.

5. Yamazi Y, Hirai K, Honda T. Pseudomembranous tracheobronchitis caused by methicillin resistant Staphylococcus aureus. Scand J Infect Dis 2002;34(3):211-213.

6. Machida U, Kami M, Kanda Y, Takeuchi K, Akahane M, Yamaguchi I, et al. Aspergillus tracheobronchitis after allogenic bone marrow transplantation. Bone Marrow Transplant 1999;24(10):1145-1149.

7. Strauss R, Mueller A, Wehler M, Neureiter D, Fischer E, Gramatzki $\mathrm{M}$, et al. Pseudomembranous tracheobronchitis due to Bacillus cereus. Clin Infect Dis 2001;33(5):E39-E41.
8. Deslee G, Brichet A, Lebuffe G, Copin MC, Ramon P, Marquette $\mathrm{CH}$. Obstructive fibrinous tracheal pseudomembrane. A potential fatal complication of tracheal intubation. Am J Respir Crit Care Med 2000;162(3 Pt 1):1169-1171.

9. Koch WM, McDonald GA. Stevens-Johnson Syndrome with subglottic laryngeal obstruction. Arch Otolaryngol Head Neck Surg 1989; 115(11):1381-1383.

10. Hubbard GB, Langlinais PC, Shimazu T, Okerberg CV, Mason AD, Pruitt BA Jr. The morphology of smoke inhalation in sheep. J Trauma 1991;31(11):1477-1486.

11. Stephens DS, Walker DH, Schaffner W, Kaplowitz LG, Brashear HR, Roberts R, Spickard WA Jr. Pseudodiphtheria: prominent pharyngeal membrane associated with fatal paraquat ingestion. Ann Intern Med 1981;94(2):202-204.

12. Herny MT, Davidson LA, Cooke NJ. Tracheobronchial involvement with Crohn's disease. Eur J Gastroenterol Hepatol 2001;13(12): 1495-1497. 